\title{
HABLEMOS DEL ACCESO ABIERTO
}

Marcela Pulido Mora ${ }^{1}$

\section{Resumen:}

Hablemos del movimiento Open Access, a partir de una reflexión conceptual que nos permita comprender que los esfuerzos sí redundan en el desarrollo de la investigación y el conocimiento, para lograr resultados encaminados a la publicación y la divulgación. Estas dos generan la visibilidad con impacto nacional e internacional y nos ofrecen la posibilidad de acceder al conocimiento académico y científico sin restricción por medio de internet.

Palabras clave: acceso a la información, divulgación científica, difusión de conocimientos.

\section{LET'S TALK ABOUT THE OPEN ACCESS MOVEMENT}

\begin{abstract}
:
Let's talk about the Open Access movement, in a conceptual reflection that allows us to understand that the efforts do result in the development of research and knowledge, to achieve results for the publication and dissemination. Those ones generate visibility with national and international impact and give us the possibility of accessing to academic and scientific knowledge without restriction on the Internet.
\end{abstract}

Keywords: access to information, science popularization, dissemination of knowledge.

(C) 2021 Fundación Universitaria Juan N. Corpas (FUJNC).

Artículo Open Access bajo la Licencia Creative Commons Atribución-NoComercial-Compartirlgual 4.0 Internacional.

DOI: 10.26752/revistaparadigmassh.v3i1.599

\footnotetext{
${ }^{1}$ Bibliotecóloga y especialista en Gerencia de Mercadeo de la Universidad de La Salle. Jefe de biblioteca en el Centro de Información y Consulta de la Fundación Universitaria Juan N. Corpas. Bogotá, D.C., Colombia. ORCID 0000-00019667-2239. Autor de correspondencia: marcela.pulido@juanncorpas.edu.co
} 
Hablemos del acceso abierto con una reflexión conceptual inicial para comprender que los esfuerzos sí redundan en el desarrollo de la investigación y el conocimiento. Inmersas en la cultura de la investigación, las instituciones de educación superior trabajan entre políticas, declaraciones, consorcios, tecnología, derechos de autor, gestión de recursos, entre otros, para lograr resultados encaminados a la publicación y divulgación que tiende a la visibilidad con impacto nacional e internacional. Sin embargo, el camino no ha sido fácil.

Open Access es un movimiento que se consolida en el año 2002 con la Iniciativa de Acceso Abierto de Budapest (BOAl, por sus siglas en inglés), como resultado de la reunión internacional liderada por el Instituto para un Sociedad Abierta (Open Society Institute OSI). Dicho encuentro tuvoe la finalidad de acelerar el progreso de la investigación en todas las áreas del conocimiento, con la convicción de abrir completamente el acceso en internet de la literatura científica y eliminar las barreras de uso. Lo anterior respeta los derechos de autor y gestiona la declaración de Budapest como la base de dicha iniciativa.

Por acceso abierto nos referimos a su disponibilidad gratuita en la internet pública, que permite a cualquier usuario leer, descargar, copiar, distribuir, imprimir, buscar o vincular a los textos completos de estos artículos y rastrearlos, para utilizarlos en cualquier fin lícito, sin barreras financieras, legales o técnicas distintas de las inseparables del acceso a internet. La única restricción sobre la reproducción y distribución y la única función de los derechos de autor en este dominio debería ser dar a los autores el control sobre la integridad de su trabajo y el derecho a ser reconocidos y citados debidamente. (Budapest Open Access Initiative, 2002).

Para estimular el debate dentro de la comunidad de investigación biomédica sobre cómo proceder hacia el objetivo generalizado de proporcionar acceso abierto a la literatura científca, se realizó una reunión en el Instituto Médico Howard Hughes en Chevy Chase, Maryland, lo que tuvo como resultado la declaración de Bethesda en el año 2003. Al ratificar los prin- cipios para acordar las rutas de desarrollo entre las organizaciones que fomentan y apoyan la investigación científica, los científicos que generan los resultados de la investigación, los editores que facilitan la revisión por pares y la distribución de los resultados de la investigación, adoptan y promueven la transición rápida y eficaz a la publicación de acceso abierto (Bethesda Statement on Open Access Publishing, 2003).

A finales del año 2003, se lleva a cabo la declaración de Berlín (Berlin Declaration on Open Access to Knowledge in the Sciences and Humanities) para promover la internet como un instrumento base del conocimiento científico global y la reflexión humana. En este encuentro se especificaron las medidas que deben ser tomadas por los responsables de la formulación de políticas de investigación, las instituciones de investigación, las agencias de financiación, las bibliotecas, los archivos y los museos (Max-Planck-Gesellschaft, 2003).

En 2005, los participantes del IX Congreso Mundial de Bibliotecas e Información en Salud, Compromiso con la Equidad, en Salvador (Bahía, Brasil) exponen dos declaraciones sobre el acceso al conocimiento. La primera, la Declaración de Salvador, Compromiso con la Equidad, solicita a los gobiernos la promoción del acceso equitativo y abierto. La segunda, la Declaración de Salvador sobre Acceso Abierto: la Perspectiva del Mundo en Desarrollo, pide a los gobiernos que exijan el acceso abierto a la investigación financiada con fondos públicos (Bireme et al., 2005).

Sucesivamente, diversas entidades y gobiernos fueron asumiendo estos manifiestos iniciales para permitir el progreso hacia la comprensión global del acceso abierto, con infinidad de estrategias y lineamientos que abrieran las posibilidades de acceder digitalmente a contenidos derivados de la producción académica y científica sin tener ningún tipo de restricción legal ni económica (Sube \& Peek, 2008). 
En el caso latinoamericano, el acceso abierto se ha convertido en una opción esencial que ha logrado su implementación en la región sin fines comerciales. Actualmente, cuenta con la disposición internacional del conocimiento abierto sin pago, sin lucro, con un futuro no tan claro y con algunas contradicciones que demuestran que se deben desarrollar más acciones para cumplir los objetivos iniciales.

Es así como en el 2017, en México, se desarrolla la Declaración Conjunta LATINDEX-REDALYC-CLACSO-IBICT, que recomienda el uso de la licencia Creative Commons Reconocimiento-NoComercial-Compartirlgual (CC BY-NC-SA, por sus siglas en inglés) para garantizar la protección de la producción académica y científica en acceso abierto. Esto tiene como objetivo crear, compartir, mantener y preservar el conocimiento de la región (Universidad de los Andes, 2018).

Si alguien quiere hacer uso de un texto científico en acceso abierto con esta licencia, nada lo impide, puede hacerlo, solamente tiene que citarlo, compartirlo igual y no comercializar. La ciencia ha funcionado a través del tiempo utilizando los textos e ideas de los otros científicos, cuyo reflejo son las referencias citadas, porque el conocimiento es una construcción social en beneficio de la humanidad; Sin duda, con esta declaración contribuimos a conformar, mantener y proteger nuestro ecosistema latinoamericano de Acceso Abierto. (Universidad de los Andes, 2018)

Así que hoy la ciencia abierta es, por su la naturaleza, una plataforma para el diálogo, la cual fomenta más intercambios y estimula a los investigadores a adaptar sus prácticas de publicación y difusión. Lo anterior les permitirá reducir los costes, la mejora de los contenidos académicos y propiciar una mayor circulación y generación del conocimiento (Antunes et al., 2020).
Contamos con un sinnúmero de prácticas y habilidades en el proceso científico y en la producción del conocimiento basados en la cooperación y en las tecnologías de la información. Así, de forma transparente y accesible, abrimos redes de colaboración y permitimos la reproductibilidad, la inclusión y la participación en la investigación con rigor y responsabilidad. Empero, también se requieren nuevos modelos de financiación que permitan el desarrollo equitativo y autosostenible.

En Colombia, tenemos un Sistema Nacional de Ciencia, Tecnología e Innovación para desarrollar los componentes de la ciencia abierta, compuesto por el Ministerio de Ciencia, Tecnología e Innovación (MinCiencias), el Ministerio de Educación Nacional (MEN), las instituciones de educación superior colombianas (públicas y privadas), institutos, centros de investigación y otras organizaciones que manifiestan un compromiso expreso en el fomento a la producción del conocimiento en acceso abierto, los cuales participan con sus repositorios institucionales. Tal es el caso del actual Nodo Nacional de la ${ }^{2}$ Referencia, que a partir del año 2020 cosecha nuestros repositorios institucionales a fin de ampliar los documentos disponibles.

Finalmente, cabe mencionar que el movimiento Open Access impacta positivamente en beneficio de la sociedad, al darnos la posibilidad de acceder al conocimiento académico y científico sin restricción en la internet. Así mismo, los autores cuentan con vías legales para compartir su producción intelectual para potenciar la difusión de su trabajo y contar con reconocimiento. Sin embargo, debemos avanzar en la generación de nuevos y mejores modelos de negocio sustentables y equitativos.

${ }^{2}$ La Referencia, da visibilidad a la producción científica de las instituciones de educación superior e investigación de América Latina, además de que promueve el acceso abierto y gratuito al texto completo, con especial énfasis en los resultados financiados con fondos públicos. 


\section{Referencias Bibliográficas}

1. Antunes, M. L., Sanches, T., \& Lopes, C. A. (2020). Publicar en el ecosistema de la ciencia abierta. Cuadernos de Documentación Multimedia, 31, 1-11. http://dx.doi.org/10.5209/cdmu.71449

2. Berlin Declaration on Open Access to Knowledge in the Sciences and Humanities (22 de octubre de 2003). Open Access. Max-Planck-Gesellschaft. https://openaccess.mpg.de/Berlin-Declaration

3. Bethesda Statement on Open Access Publishing. (20 de junio de 2003). https://dash.harvard.edu/bitstream/handle/1/4725199/Suber_bethesda.htm?sequence=3\&isAllowed $=\mathrm{y}$

4. Bireme, OPS, OMS, Centro Latinoamericano y del Caribe de Información en Ciencias de la Salud, Ministerio de Salud de Brasil, International Federation Library Association. (2005). $9^{\circ}$ Congreso Mundial de Información en Salud y Bibliotecas y $7^{\circ}$ CRICS. http://www.icml9.org/channel.php?lang=es\&channel=87

5. Budapest Open Access Initiative. (2002). https://www.budapestopenaccessinitiative.org/ Sube, P. \& Peek, R. (2008). Declarations in support of OA. Wikipedia. http://oad.simmons.edu/oadwiki/Declarations_in_support_of_OA

6. Universidad de los Andes. (2018). Declaración de México a favor del Ecosistema Latinoamericano de acceso abierto no comercial. Educere, 22(71), 201-204. Recuperado el 03 de julio de 2021, de https://www.redalyc.org/articulo.oa?id=35656002017 